\title{
Adult Mouse Motor Units Develop Almost All of Their Force in the Subprimary Range: A New All-or-None Strategy for Force Recruitment?
}

\author{
Marin Manuel and C. J. Heckman \\ Department of Physiology, Northwestern University, Feinberg School of Medicine, Chicago, Illinois 60611
}

Classical studies of the mammalian neuromuscular system have shown an impressive adaptation match between the intrinsic properties of motoneurons and the contractile properties of their motor units. In these studies, the rate at which motoneurons start to fire repetitively corresponds to the rate at which individual twitches start to sum, and the firing rate increases linearly with the amount of excitation ("primary range") up to the point where the motor unit develops its maximal force. This allows for the gradation of the force produced by a motor unit by rate modulation. In adult mouse motoneurons, however, we recently described a regime of firing ("subprimary range") that appears at lower excitation than what is required for the primary range, a finding that might challenge the classical conception. To investigate the force production of mouse motor units, we simultaneously recorded, for the first time, the motoneuron discharge elicited by intracellular ramps of current and the force developed by its motor unit. We showed that the motor unit developed nearly its maximal force during the subprimary range. This was found to be the case regardless of the input resistance of the motoneuron, the contraction speed, or the tetanic force of the motor unit. Our work suggests that force modulation in small mammals mainly relies on the number of motor units that are recruited rather than on rate modulation of individual motor units.

\section{Introduction}

It is thought that, in any neural system, two elements communicating with each other are "tuned" in such a way that the transfer of information can be maximally efficient. The neuromuscular system is particularly well suited to test such ideas, because each muscle fiber is connected to only one motoneuron, and both elements are relatively easy to record. Hence, it is expected that the discharge of a motoneuron be constrained to the frequencies relevant to the production of physiologically meaningful forces, and, vice versa, that the contractile properties of muscle fibers be adapted to the range of firing produced by the motoneuron to which they are connected. In support of this hypothesis, it has been shown both in cat (Huizar et al., 1977; Dum and Kennedy, 1980; Zengel et al., 1985; Cope et al., 1986) and rat (Bakels and Kernell, 1993a,b) neuromuscular systems that the duration of the afterhyperpolarization (AHP), which follows each spike of a motoneuron and is essential in controlling its discharge (Manuel et al., 2006; Stauffer et al., 2007), is tuned to the duration of the

\footnotetext{
Received June 8, 2011; revised July 29, 2011; accepted Aug. 29, 2011.

Author contributions: M.M. and C.J.H. designed research; M.M. performed research; M.M. analyzed data; M.M. and C.J.H. wrote the paper.

M.M. was supported by the Fondation pour la Recherche Médicale and the Milton Safenowitz PostDoctoral Fellowship for ALS Research (ALS Association). This work was financed by NIH NINDS Grant NS05462 and NS034382. We thank Dr. Jack Miller and Prof. Donald McCrimmon for their technical assistance, and Dr. Daniel Zytnicki for his comments on this manuscript.

The authors declare no competing financial interests.

Correspondence should be addressed to Marin Manuel, Department of Physiology, Northwestern University, Feinberg School of Medicine, 303 East Chicago Avenue, Chicago, IL 60611. E-mail: marin.manuel@neurobio.org. DOI:10.1523/JNEUROSCI.2893-11.2011

Copyright $\odot 2011$ the authors $\quad 0270-6474 / 11 / 3115188-07 \$ 15.00 / 0$
}

twitch of the muscle fibers that it innervates (Burke, 1980, 1981; Kernell, 2006).

Only a few electrophysiological investigations of spinal motoneurons have been performed so far in anesthetized adult mice (Huizar et al., 1975; Alstermark and Ogawa, 2004; Manuel et al., 2009; Meehan et al., 2010). Recently, we have demonstrated that the firing properties of mouse motoneurons differ from the cat's in one key aspect: although there is a range of input for which the firing frequency increases linearly and regularly with the amount of injected current (i.e., a "primary range," PR), it is preceded by a "subprimary range" (SPR) where the $I-F$ curve is very steep and the discharge is very irregular because of fast subthreshold oscillations that generate interspike intervals longer than the duration of the AHP (Manuel et al., 2009; Iglesias et al., 2011).

This difference between cat and mouse motoneurons raises important questions with respect to the match between the firing and the contractile properties of a motor unit. Because, in the SPR, the AHP does not appear to be the only factor controlling the firing frequency, is the contraction of a motor unit still "speed matched" to its motoneuron? Does the force gradation happen in the SPR or in the PR, which is the case in cat motor units?

To answer these questions, we extended our preparation to obtain, for the first time in mice, stable intracellular recordings in unparalyzed animals despite the contraction of the muscles. This allowed us to record simultaneously the motoneuron discharge and the force developed by its motor unit. Unexpectedly, the contractile force increased steeply in the SPR, and most of the force was already recruited at the transition between the SPR and the $\mathrm{PR}$, regardless of the contraction speed of the motor unit. This suggests that force modulation in small mammals mainly relies 

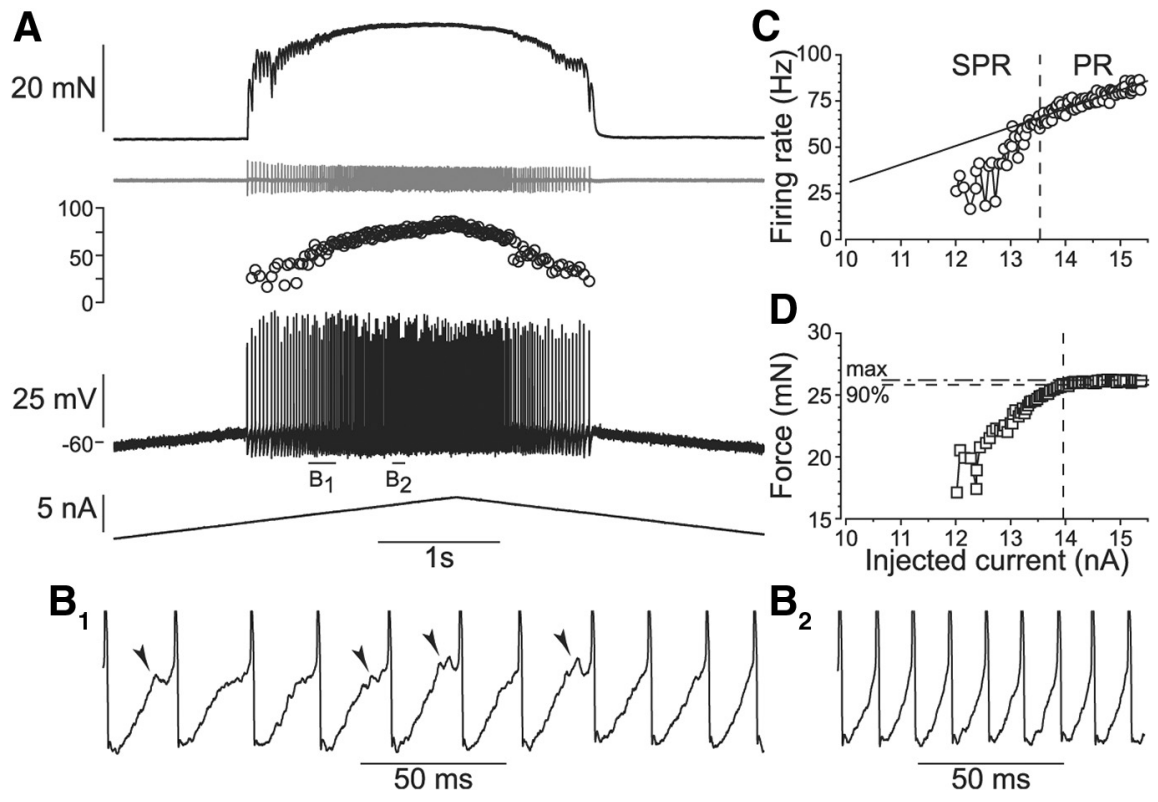

Figure 1. Force profile of a fast motor unit. $A$, From top to bottom: force, surface EMG, frequencygram, membrane potential, injected current. $\boldsymbol{B}_{1}, \boldsymbol{B}_{2}$, Magnifications of the regions indicated in $\boldsymbol{A}$. Arrowheads indicate the fast subthreshold oscillations characteristic of the SPR. $C$, Instantaneous firing frequency versus the intensity of the injected current. Vertical dashed line indicates the limit between SPR and PR. D, Peak force versus the intensity of current. The maximal force developed by the motor unit ("max") and $90 \%$ of the force range are indicated. The vertical dashed line represents $I_{90 \%}$.

on motor unit recruitment rather than on rate modulation of individual motor units.

\section{Materials and Methods}

Experiments were performed on 16 female adult CD1 mice aged between 34 and $37 \mathrm{~d}$ old (Harlan Laboratories). Experiments were performed under full approval from the Northwestern University Animal Care and Use Committee. At the onset of experiments, atropine $(0.20 \mathrm{mg} / \mathrm{kg}$, MedPharmex) was given subcutaneously to prevent salivation. Ten minutes later, the anesthesia was induced with an intraperitoneal injection of sodium pentobarbital (70 mg/kg, Nembutal, Ovation Pharmaceuticals). A tracheotomy was performed, and the mouse was artificially ventilated with pure oxygen (SAR-830/P ventilator, $\mathrm{CWE}$ ). The end-tidal $\mathrm{P}_{\mathrm{CO}_{2}}$ was maintained around $4 \%$ (MicroCapstar, CWE). The heart rate was monitored, and the central temperature was kept at $38^{\circ} \mathrm{C}$ using an infrared heating lamp. A catheter was introduced in the external jugular vein, allowing for supplementary anesthesia by intravenous injections $(6 \mathrm{mg} /$ $\mathrm{kg}$ ) whenever necessary (usually every 10-20 min). The adequacy of anesthesia was assessed on the lack of noxious reflexes and on the stability of the heart rate (usually $400-500$ beats per minute) and end-tidal $\mathrm{P}_{\mathrm{CO}_{2}}$. Low intravenous infusion $(50 \mu \mathrm{l} / \mathrm{h})$ of a $4 \%$ glucose solution containing $\mathrm{NaHCO}_{3}(1 \%)$ and hetastarch (14\%, Hospira) helped to maintain the physiological parameters. All of the branches of the sciatic nerve except for the ones innervating the triceps surae (TS), were dissected, cut distally, and mounted on bipolar electrodes for stimulation. The branches innervating the TS were left in continuity and mounted on a monopolar electrode. To obtain stable intracellular recordings, it was paramount to avoid any movement of the spinal cord while the muscles were contracting. The preparation was therefore secured in several points. The vertebral column was immobilized with two pairs of horizontal bars (Cunningham Spinal Adaptor, Stoelting) applied on the Th13 and L2 vertebral bodies, and the L3-L4 spinal segments were exposed by a laminectomy at the Th13-L1 level. The sacrum was immobilized by a separate clamp, while the hindlimb was maintained in a fixed, $90^{\circ}$ position, by a third clamp immobilizing the tibia. The tissues of the hindlimb and the spinal cord were covered with pools of mineral oil. The tendon of the TS was dissected free and securely attached to an isometric force transducer (BG-100, Kulite). At the end of the experiments, animals were killed with a lethal intravenous injection of pentobarbital.
Intracellular recordings were performed using glass microelectrodes (tip diameter 1.0-1.5 $\mu \mathrm{m}$ ) filled with $\mathrm{KCl} 3 \mathrm{M}$ (resistance $8-15 \mathrm{M} \Omega$ ). Recordings were made using an Axoclamp 2B amplifier (Molecular Devices), a micro1401 interface, and the Spike2 software (CED). Motoneurons were identified by antidromic stimulation of their axons. All motoneurons retained for analysis had a resting membrane potential more hyperpolarized than $-50 \mathrm{mV}$ and an overshooting action potential larger than $65 \mathrm{mV}$. After penetration, a series of small-amplitude ( -3 to $+3 \mathrm{nA}), 500 \mathrm{~ms}$ square current pulses were used to plot the $I-V$ (current-voltage) relationship. The characteristics of action potentials were measured on spikes elicited by short intracellular depolarizing pulses of current $(5 \mathrm{nA}, 0.5 \mathrm{~ms})$ repeated at the frequency of $2 \mathrm{~Hz}$. We averaged $\sim 20$ successive records. In particular, we measured the AHP total duration by finding, using a custom software, the point at which the AHP had repolarized by $99 \%$, and then visually checking and eventually correcting the accuracy of this measurement (Manuel et al., 2009).

At the beginning of the experiment, the initial length of the muscle was set to the length at which the ankle was flexed at $90^{\circ}$. This length was then left constant for the duration of the experiment. The force signal was amplified (typically 200 or $500 \times$ ) and filtered at $4 \mathrm{kHz}$ (CyberAmp, Molecular Devices). The twitch properties were recorded in response to single action potentials, and their characteristics measured off-line after averaging at least five sweeps. We measured the twitch amplitude, the contraction time (i.e., the time between the onset of the twitch and the time at which it reached its maximum), the twitch half decay time, and the total duration (i.e., the time between the onset of the twitch and point at which it had declined to $99 \%$ of its maximum value), using the same automated approach as described above. We also recorded the force profile produced by the repetitive firing obtained in response to a slow triangular ramp of current $(0.5-2.0 \mathrm{nA} / \mathrm{s})$. This allowed us to plot the force-frequency curve of the motor unit, which was, in all cases, sigmoidal. This curve was used to measure the tetanic force as the value reached on the upper plateau of the sigmoid, and the tetanic fusion frequency (TFF) was estimated as the frequency for which the force was equal to $99 \%$ of the tetanic force. We then estimated the amount of current required to recruit most of the force. Given that the smallest force developed by a given motor unit is the force generated by a single twitch, we measured the amount of current necessary to recruit $90 \%$ of the working range of the motor unit, i.e., between the lower bound of the single twitch amplitude and the tetanic force. Ninety percent of the force range is then defined by the following quantity: twitch force $+0.9 \times$ (tetanic force twitch force). Accordingly, we noted $I_{90 \%}$, the current necessary to reach this force. Surface EMG (i.e., single motor unit compound action potential) was recorded at the same time using fine stainless steel wires inserted underneath the TS fascia and a Grass P5 amplifier (signal amplified $100 \times$, and filtered at $10 \mathrm{kHz}$ ).

\section{Results}

\section{Force is maximal near recruitment}

We were able to study, for the first time, the recruitment and force gradation of single mouse motor units in 12 motoneurons, because our preparation allowed us to obtain stable intracellular recordings and simultaneously record the force output of the motor unit.

A typical example is shown in Figure 1. The force developed by the motor unit displayed an unexpected profile: twitch summation occurred during the first interspike intervals, and conse- 
quently, the force output increased very sharply and quickly reached a plateau value. This steep rise in force occurred even though the discharge started in the SPR, a regime of firing that we recently described in mouse motoneurons (Manuel et al., 2009). The SPR is characterized by high-frequency, subthreshold oscillations following the AHP (Fig. $1 B_{1}$, arrowheads), creating long interspike intervals, often outlasting the AHP. It is thus apparent that, despite the low initial firing rate, individual twitches are able to sum during the SPR. At higher current intensity, the firing entered the PR, where the interspike intervals became more regular and the oscillations disappeared (Fig. $1 B_{2}$ ). In the $\mathrm{PR}$, the firing rate increased linearly with the injected current, yet the force remained at a steady value. The force followed a similar profile on the descending branch of the ramp of current, falling down to baseline steeply at the end of the firing.

To compare the evolution of the firing frequency and the force with respect to the amount of injected current, we plotted the instantaneous frequency versus the current intensity on the ascending ramp (Fig. 1C, $I-F$ curve) and the peak force after each spike versus the current intensity (Fig. $1 D$, current-force curve). The $I-F$ curve illustrates that the discharge is initially greatly variable in the SPR but becomes much less variable when the PR is reached. In this motoneuron, the current at the transition between the SPR and the PR $\left(I_{\text {trans }}\right)$ was equal to $13.5 \mathrm{nA}$ (Fig. $1 C$, vertical line). On the current-force curve (Fig. $1 D$ ), we measured the current intensity at which $90 \%$ of the force range was recruited. This current intensity $\left(I_{90 \%}\right.$, Fig. $1 D$, vertical line) was equal to $13.9 \mathrm{nA}$ in this motoneuron, which is very close to the transition between SPR and PR.

In the motoneuron shown in Figure 1, the firing transitioned from the SPR to the PR at a relatively high frequency $(66 \mathrm{~Hz})$, which might explain why the motor unit had almost developed its maximal force. However, Figure 2 shows another motoneuron that had a higher input resistance ( 6.4 vs $1.7 \mathrm{M} \Omega$ ) and a slower contraction time (34 vs $12 \mathrm{~ms}$ ). Similar to the previous example, this motoneuron started to fire in the SPR, and transitioned to the PR for a current intensity $I_{\text {trans }}$ equal to $2.1 \mathrm{nA}$ and a firing rate of $33 \mathrm{~Hz}$ (Fig. 2B, vertical line). Examination of the force profile revealed that, once again, the force increased very steeply just after the onset of firing and quickly reached a plateau where it did not increase anymore, despite a continued linear increase in firing rate through the PR (Fig. $2 B$, solid line). The current for which $90 \%$ of the force range was developed, $I_{90 \%}$, was equal to $1.9 \mathrm{nA}$ in this motoneuron.

Among the 12 motor units in which we tested the ramps of current, we injected enough current to reach the PR (and thereby measure the transition current) in nine. In each motoneuron, we carefully estimated the transition current as the point where the $I-F$ curve deviates from the linear PR. The value of $I_{\text {trans }}$ was dramatically different among motoneurons, and ranged from 2.1 to $13.5 \mathrm{nA}$ (mean $\pm \mathrm{SD}, 6.4 \pm 3.8 \mathrm{nA}, N=9$ ). Despite this wide scatter, the current necessary to reach $90 \%$ of the force range, $I_{90 \%}$ $(1.9-13.9 \mathrm{nA} ; 6.4 \pm 3.6 \mathrm{nA}, N=9)$, was not statistically different from $I_{\text {trans }}$ (two-tailed paired Student's $t$ test, $p=0.26$ ), and the two values were closely correlated as shown in Figure $3(r=0.99$, $p<0.0001)$. This demonstrates the novel result that most of the force gradation occurs in the SPR, and that almost no additional force is produced as the frequency increases in the PR.

However, the "width" of the SPR is small. It ranged from 0.5 to $2.2 \mathrm{nA}(1.1 \pm 0.6 \mathrm{nA}, N=9)$. Therefore, once the motoneuron was recruited, only small amounts of extra current were generally required to reach the PR. Compared with the amount of excitation required to recruit the motoneurons (onset current), an extra 9-62\% of additional current (30 \pm $20 \%, N=9$ ) is sufficient to recruit $90 \%$ of the force of the motor unit.

\section{Motoneurons are faster than their muscle fibers}

Three features of mouse motor units can explain how most of the force can be developed in such a small range of excitation. First, the duration of the AHP is shorter than the duration of the twitch. Figure $4, A$ and $B$, illustrates two motor units with different twitch responses: the example shown in Figure $4 A$ had a large twitch (peak amplitude $10.4 \mathrm{mN}$ ), but of short duration (contraction time $12 \mathrm{~ms}$, total duration $52 \mathrm{~ms}$ ), whereas the other example (Fig. $4 B$ ) had a much smaller $(1.0 \mathrm{mN}$ ) and longer-lasting (contraction time $33 \mathrm{~ms}$, duration $139 \mathrm{~ms}$ ) twitch. It is apparent in these two examples that the AHP duration is shorter than the twitch duration. In a sample of 33 motor units, the total twitch duration was strongly correlated to the total duration of the AHP $(r=0.89, p<0.0001$, Fig. $4 C)$. Total twitch duration, however, averaged 30\% longer than AHP duration (see Fig. $4 D ; 53 \pm 31 \mathrm{~ms}$ vs $39 \pm 15 \mathrm{~ms}, N=33$, respectively; one-tailed paired Student's $t$ test, $p=0.005)$. The longer twitch duration allows individual twitches to sum in the subprimary firing range, where interspike intervals can be longer than the duration of the AHP (Manuel et al., 2009).

\section{Steep force onset through low neuronal excitability}

Second, the motoneuron firing starts at low frequency but increases very steeply during the SPR. This is because mouse motoneurons are relatively hypoexcitable (Iglesias et al., 2011). This state delays the firing onset and creates the subthreshold oscillations and the SPR of firing. Indeed, suppose that mouse motoneurons did not exhibit this low excitability and that (like cat motoneurons) they would then start to fire at a frequency corresponding to the inverse of their AHP duration. Under these assumptions, and based on their $I-F$ curve, we can estimate the current intensity at the onset of firing and the range of current 


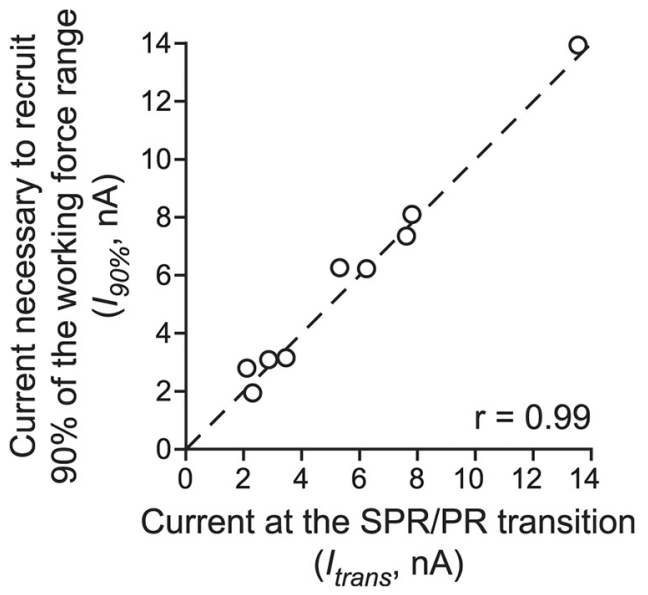

Figure 3. Most of the force is developed in the SPR. Current necessary to recruit $90 \%$ of the force range $\left(I_{90 \%}\right)$ versus current at the SPR/PR transition $\left(I_{\text {trans }}\right)$ is plotted. There was a highly significant correlation between the two parameters $(r=0.99, p<0.0001)$. The dashed line represents the identity line.
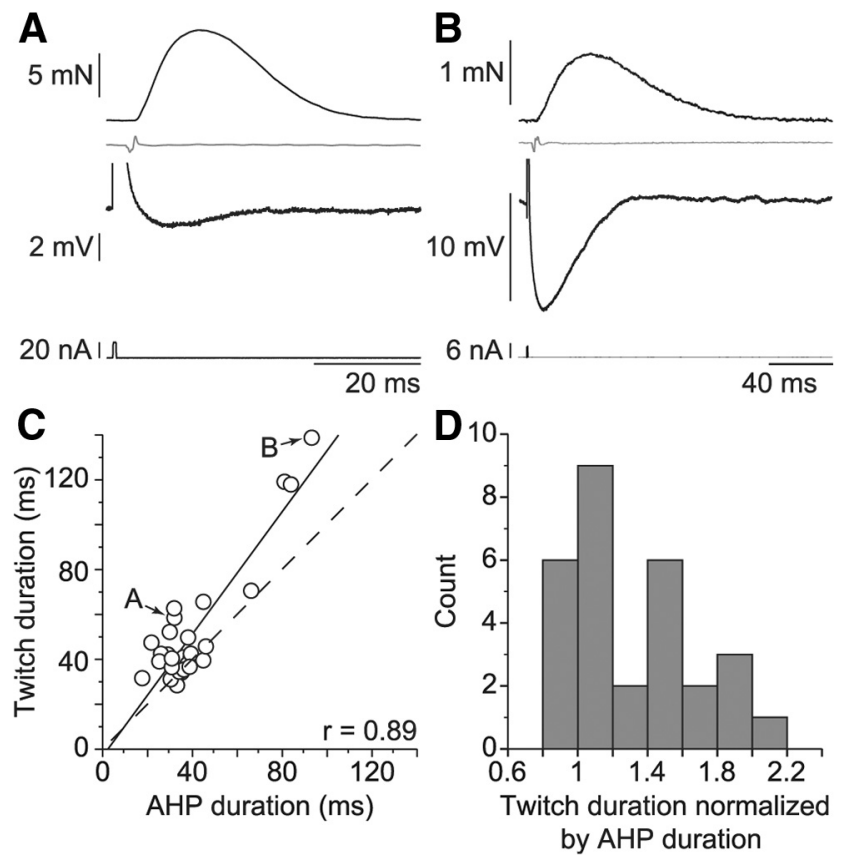

Figure 4. Motoneurons are faster than their muscle fibers. $\boldsymbol{A}$, Single motor unit twitch (average of 10 sweeps) from the same motor unit as in Figure 1. B, Single twitch (average of 10 sweeps) from the same motor unit as in Figure 2. C, Twitch duration versus AHP duration. The solid line represents the best linear fit $(r=0.89, p<0.0001)$, and the dashed line represents the identity line. $\boldsymbol{D}$, Histogram of the ratio twitch duration over AHP duration. Range $0.9-2.2$, mean \pm SD $1.3 \pm 0.3 ; N=33$.

needed to increase the firing rate from onset frequency to the tetanic fusion frequency (Fig. 5A). Without subthreshold oscillations, the current onset would be much lower $(3.4 \pm 3.0 \mathrm{nA}$ vs $4.6 \pm 3.4 \mathrm{nA} ; N=9$; one-tailed paired Student's $t$ test $p=0.001$ ). Consequently, the range of excitation over which frequency would increase to reach the tetanic fusion frequency of the motor unit would be larger (1.3-3.8 nA; $2.1 \pm 0.9 \mathrm{nA} ; N=9$; one-tailed paired Student's $t$ test $p=0.002$ ) compared with the width of the SPR above. Therefore, if mouse motor units did not have subthreshold oscillations, force gradation would be much more progressive.

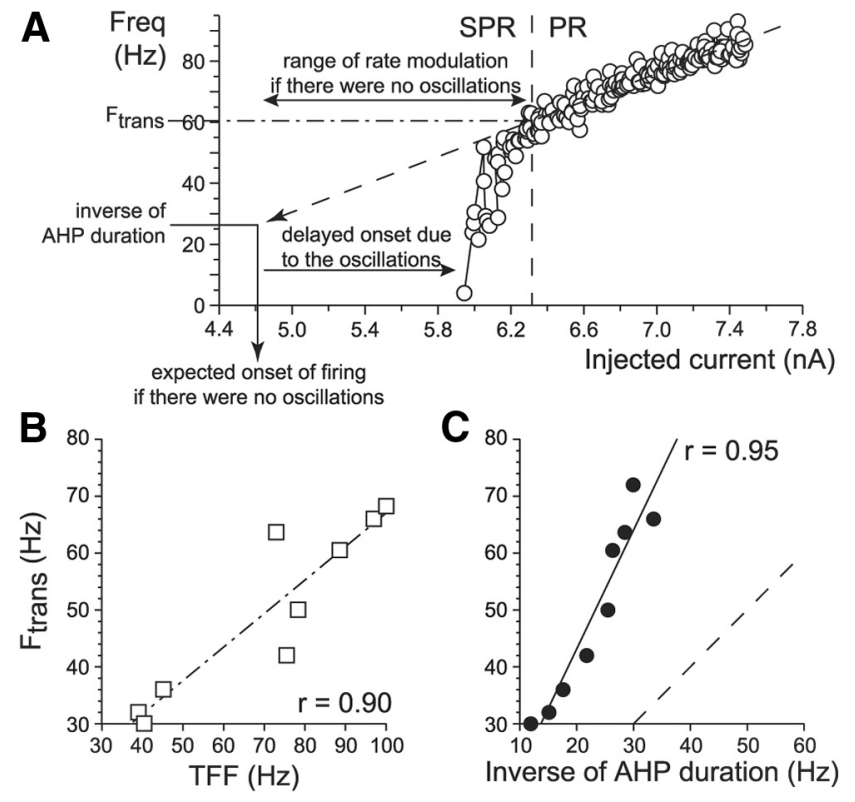

Figure 5. The SPR/PR transition is matched to the contractile and electrophysiological properties of the motor unit. $\boldsymbol{A}$, Schema illustrating how the current-frequency relationship would be modified if mouse motoneurons did not have subthreshold oscillations and an SPR of firing. $\boldsymbol{B}$, Frequency at the SPR/PR transition $\left(F_{\text {trans }}\right)$ versus TFF. Dashed line is the best linear fit $(r=$ $0.90, p=0.0005) . C, F_{\text {trans }}$ was strongly correlated to the inverse of the AHP duration $(r=0.95$, $p<0.0001$ ), but was always greater (dashed line represents the identity line).

\section{The transition frequency is precisely tuned}

Third, the firing rate reached at the transition SPR/PR is tuned to the TFF of the motor unit, allowing thereby a high, almost tetanic level of force at (or around) the start of the PR. The TFF showed a wide scatter and ranged from 44 to $100 \mathrm{~Hz}(70 \pm 24 \mathrm{~Hz} ; N=9)$. The firing frequency reached at the SPR/PR transition, $F_{\text {trans }}$, was also widely spread among motoneurons from 32 to $75 \mathrm{~Hz}$ ( $55 \pm$ $15 \mathrm{~Hz} ; N=9$ ). Despite their wide range, the firing frequency at the SPR/PR transition was surprisingly well correlated to the TFF $(r=0.90, p=0.001$; Fig. $5 B) . F_{\text {trans }}$ was also correlated to the kinetics of the twitch: motor units with a short twitch all had a high $F_{\text {trans }}$, above $50 \mathrm{~Hz}$, whereas motor units with slower twitches had a much lower $F_{\text {trans }}(r=-0.78, p<0.005)$. This is in keeping with the fact that the highest TFFs are observed in the motor units that display the shortest twitch duration $(r=-0.85$; $p=0.004)$. Our results suggest therefore that the transition frequency is precisely controlled in motor units of different size. $F_{\text {trans }}$ was indeed strongly correlated to the input resistance of the motoneurons $(r=-0.92, p=0.001)$. We found that the AHP duration plays the key role. As shown in Figure $5 C, F_{\text {trans }}$ was proportional to the inverse of AHP duration $(r=0.95, p<$ 0.0001): the motoneurons with a short AHP had a higher transition frequency than those with a longer AHP. $F_{\text {trans }}$ was also correlated to the AHP amplitude $(r=-0.91, p=0.002$; data not shown). This is in keeping with our previous finding that showed that a motoneuron transitions from the SPR to the PR when its firing rate becomes too high for the AHP to relax completely (Iglesias et al., 2011).

\section{Size principle in mouse motor units}

Despite the low excitability of mouse motoneurons, their recruitment is still likely to follow the size principle. Indeed, in our sample of 33 motoneurons, the input resistances were spread over an almost 10-fold range (1.0-6.4 M $2,2.7 \pm 1.5 \mathrm{M} \Omega, N=33$; 

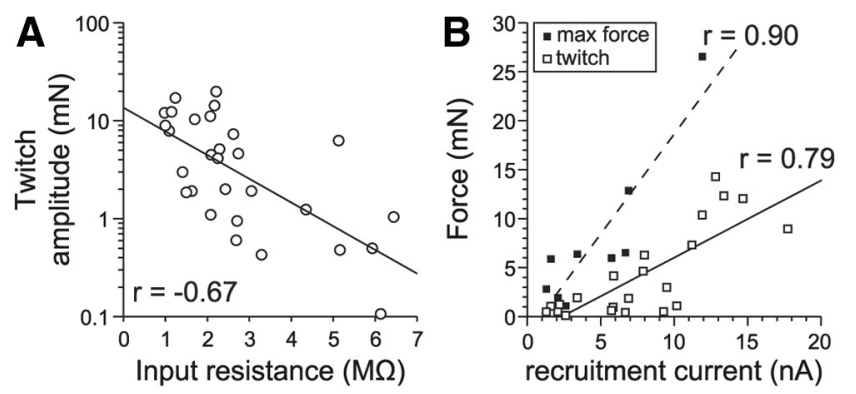

Figure 6. Size principle in mouse motoneurons. $\boldsymbol{A}$, Semilogarithmic plot of the twitch peak amplitude versus the input resistance in our sample of 33 motor units. There was a strong correlation between these two properties ( $r=-0.67, p<0.0001)$. The solid line represents the best linear fit. $\boldsymbol{B}$, The peak twitch force (empty squares) is strongly correlated to the recruitment current of the motor unit ( $r=0.79, p<0.0001)$. The tetanic force (filled squares) also strongly depended on the recruitment current $(r=0.90, p=0.0009)$.

Fig. $6 A$ ), which created, in turn, a wide range of recruitment currents (from 1.3 to $18.6 \mathrm{nA}, 8.0 \pm 5.0 \mathrm{nA}, N=33$; Fig. $6 B$ ). This wide range allows the progressive recruitment of more and more units as the synaptic drive to the motor pool increases. Furthermore, we found that the twitch amplitude, contraction time, and twitch total duration of a motor unit were strongly correlated to the electrophysiological parameters of their motoneuron. Figure $6 \mathrm{~A}$ shows that smaller motoneurons (with a higher input resistance) had systematically smaller twitch amplitudes than bigger motoneurons did $(r=-0.67, p<0.0001)$. Accordingly, Figure $6 B$ shows that the units with a small twitch amplitude (or a small tetanic force) have a low recruitment threshold, whereas units producing large forces are recruited at higher levels of excitation ( $r=0.79$ for the twitch amplitude, $r=0.90$ for the tetanic force, $p<0.001$ in both cases). We also observed that the smaller the motoneuron, the slower its motor unit, and that contraction time gets faster as the input resistance decreases $(r=0.70, p<0.0001)$. Altogether, the almost 10-fold range of input resistance, as well as the correlation between force and recruitment threshold, indicates that mouse motoneurons most likely respect Henneman's size principle. That is, small motoneurons, which develop little force, are recruited first, while larger, more powerful motor units require greater excitation to reach threshold.

\section{Discussion}

We present here, for the first time in mice, concurrent investigation of both the electrical properties of a motoneuron and the mechanical properties of its muscle fibers. We show that mouse motor units develop almost all of their force in the SPR, even before the start of the PR. This is possible because (1) mouse motoneurons are faster than their muscle fibers, allowing individual twitches to sum even in the range of frequencies corresponding to the SPR; (2) the relative hypoexcitability of mouse motoneurons creates an SPR where the firing frequency rises steeply; and (3) the processes controlling the SPR are adapted to the contractile properties of the motor unit in such a way that the frequency reached at the transition from the subprimary to the $\mathrm{PR}$ is close to the tetanic fusion frequency of the motor unit.

\section{Recruitment of motor units in physiological conditions}

These results have been obtained in deeply anesthetized animals and with current injected into the soma of lumbar motoneurons. In more physiological conditions, it has been shown in multiple species_-including cats (Schwindt and Crill, 1980; Conway et al., 1988; Crone et al., 1988), rats (Bennett et al., 2001; Powers and
Binder, 2003; Button et al., 2006; Turkin et al., 2010), and mice (Carlin et al., 2000; Meehan et al., 2010) — that spinal motoneurons express strong inward persistent currents (PICs) that can greatly amplify synaptic inputs (Heckman et al., 2003; Powers and Binder, 2003; Hultborn et al., 2004; Heckman et al., 2005; Manuel et al., 2007).

We have shown that the SPR and the subthreshold oscillations are due to a state of relative hypoexcitability, and increasing the excitability of the cell by increasing the sodium PIC tends to suppress the subthreshold oscillations and reduce the SPR (Iglesias et al., 2011). However, recent experiments in rats anesthetized with ketamine/xylazine (Turkin et al., 2010) and in mice anesthetized with fentanyl/midazolam (Meehan et al., 2010) showed that an SPR is present in these motoneurons despite the visibility of clear signs of PICs. Therefore, the presence of PICs in physiological conditions does not preclude the steep increase in force during the SPR in mouse motor units. Furthermore, during normal motor behavior, PICs are activated around or even below the recruitment threshold (Heckman et al., 2005). It is thus likely that, when they are recruited by synaptic excitation, the PICs of mouse motoneurons will push the firing past the SPR and therefore facilitate the development of the maximal force by the motor units as soon as they are recruited.

\section{SPR in other species}

The term "subprimary" range was initially coined to describe a regime of irregular firing at very low frequency in human motor units (Kudina and Alexeeva, 1992; Kudina, 1999). This type of firing was assumed to be caused by noise fluctuations, and the human SPR might therefore be different from the mouse SPR. Nevertheless, since our initial description of the mouse SPR (Manuel et al., 2009), it became increasingly apparent that an SPR could be observed in other species. Indeed, experiments in rats anesthetized with ketamine/xylazine showed that an SPR is also present in these motoneurons (Turkin et al., 2010). Most recordings in cat motoneurons show only a very linear $\mathrm{PR}$, but sometimes, a few spikes with a sharper slope can precede the onset of the PR (see, for example, Grillner and Udo, 1971; as well as Fig. 1C of Conway et al., 1988).

\section{A new cross-species adaptation rule for the "speed matching" concept}

In cat motor units, the duration of the AHP matches the duration of the twitch ("speed matching," Kernell, 2006) and, as reviewed by Meehan et al. (2010), both the AHP and the twitch get briefer as investigated animals get smaller, indicating an adaptation between motoneuron and muscle properties across species. Our work, however, revealed that this adaptation is not a mere size scaling. Contrary to previous studies in cats (Huizar et al., 1977; Dum and Kennedy, 1980; Zengel et al., 1985; Cope et al., 1986; Kernell, 2006), we found that the AHP was systematically shorter than the contraction time. However the AHP duration is still tuned to the contraction time of the motor unit, in that durations and contraction times were correlated (Fig. 4C). In rats, the motor unit contraction time is also slightly longer than the AHP duration of lumbar motoneurons: Bakels and Kernell (1993a,b) have shown that, on average, the AHP duration tends to be a little smaller than the twitch duration in rat motor units (14\% smaller in the tibialis anterior, Bakels and Kernell, 1993b; and see Fig. 5B of Bakels and Kernell, 1993a, for an investigation in the medial gastrocnemius). The longer twitches (relatively to the AHP duration) might thereby allow individual twitches to sum despite the 
low firing rate characteristic of the SPR, and the force to increase abruptly because of the steepness of the SPR.

To our knowledge, there are no records in the literature using the same protocol as ours to provide a direct comparison; however, the results obtained with stimulations at fixed frequencies can be compared with our results obtained with slowly rising ramps of current. As such, in the rat, given the frequencies reached at the end of the SPR $(20-60 \mathrm{~Hz})$, Turkin et al. (2010) have estimated that $\sim 60 \%$ of the maximal force would be recruited at the beginning of the PR. In cat motor units, only $10 \%$ of the force is developed at recruitment (i.e., at the beginning of the $\mathrm{PR})$, and the rest of the force is recruited by rate modulation (Kernell, 2006).

From these observations, it appears that, as one goes down in scale from large mammals to mice, the duration of individual twitches decreases, but the duration of the AHP decreases even faster, creating a situation where the twitches can sum in the SPR, which is itself more manifest in smaller animals. Consequently, the relative amount of force developed by a single motor unit at the beginning of the PR increases as one goes down in scale. This likely holds true regardless of the motor unit type. Turkin et al. (2010) have indeed shown that the shape of the SPR (slope and frequency at the SPR/PR transition) depends on electrical properties that are classically associated with the physiological type of motoneurons (input conductance and AHP amplitude). Here we show that the higher the transition frequency, the smaller the twitch duration, the AHP amplitude and duration, and the input resistance. Altogether, our work extends the classical concept of "speed matching" and points to a new cross-species adaptation rule.

\section{A new strategy for force production}

Classical experiments in cats (Kernell, 2006), primates (Palmer and Fetz, 1985), and humans (Hornby et al., 2002) have suggested that force gradation is controlled by a combination of recruitment and rate modulation. In sharp contrast, the present work shows that, in mouse motor units, most of the force is already recruited by the end of the SPR. However, the SPR is small in terms of current: compared with the amount of excitation required to recruit the motor unit, only a small amount of extra excitation is sufficient to recruit most of the force. This suggests that mouse motor units are recruited in a nearly "all-or-none" fashion and that rate modulation plays little to no role in force gradation in these small animals. The only possible strategy to increase the force output in mice is to recruit more and more motor units.

According to the "size principle" enunciated by Henneman et al. (1965), the size-related intrinsic properties (membrane resistivity, recruitment current, etc.) were found to dictate the order of recruitment of motor units (Gustafsson and Pinter, 1985). The present work suggests that this holds true in mice. Indeed, we found that the range of input resistances and, by extension, recruitment thresholds is as spread in mice as in cats, which guarantees that, given a common input to the pool of motoneurons, the smallest motor units will be recruited first, followed only at higher level of excitation by larger units. Furthermore, the units with the highest input resistance (that are therefore recruited first) are the ones that develop the smallest forces, whereas the units that develop a large force have a smaller resistance and are recruited only at higher amount of excitation.

Therefore, our results lead us to propose that a different strategy for motor control has been selected in small versus large mammals: the smaller the animal, the more it relies on recruit- ment to control its force output, instead of rate modulation. The resulting abrupt onset of the force would be a considerable advantage in animals with low inertia such as mice by assisting them in the production of strong movements in a minimal amount of time.

\section{References}

Alstermark B, Ogawa J (2004) In vivo recordings of bulbospinal excitation in adult mouse forelimb motoneurons. J Neurophysiol 92:1958-1962.

Bakels R, Kernell D (1993a) Matching between motoneurone and muscle unit properties in rat medial gastrocnemius. J Physiol 463:307-324.

Bakels R, Kernell D (1993b) Average but not continuous speed match between motoneurons and muscle units of rat tibialis anterior. J Neurophysiol 70:1300-1306.

Bennett DJ, Li Y, Siu M (2001) Plateau potentials in sacrocaudal motoneurons of chronic spinal rats, recorded in vitro. J Neurophysiol 86:19551971.

Burke R (1981) Motor units: anatomy, physiology, and functional organization. In: Handbook of physiology: the nervous system motor control, pp 345-421. Bethesda, MD: American Physiological Society.

Burke RE (1980) Motor unit types - functional specializations in motor control. Trends Neurosci 3:255-258.

Button DC, Gardiner K, Marqueste T, Gardiner PF (2006) Frequencycurrent relationships of rat hindlimb alpha-motoneurones. J Physiol 573:663-677.

Carlin KP, Jones KE, Jiang Z, Jordan LM, Brownstone RM (2000) Dendritic L-type calcium currents in mouse spinal motoneurons: implications for bistability. Eur J Neurosci 12:1635-1646.

Conway BA, Hultborn H, Kiehn O, Mintz I (1988) Plateau potentials in alpha-motoneurones induced by intravenous injection of L-dopa and clonidine in the spinal cat. J Physiol 405:369-384.

Cope TC, Bodine SC, Fournier M, Edgerton VR (1986) Soleus motor units in chronic spinal transected cats: physiological and morphological alterations. J Neurophysiol 55:1202-1220.

Crone C, Hultborn H, Kiehn O, Mazieres L, Wigström H (1988) Maintained changes in motoneuronal excitability by short-lasting synaptic inputs in the decerebrate cat. J Physiol 405:321-343.

Dum RP, Kennedy TT (1980) Physiological and histochemical characteristics of motor units in cat tibialis anterior and extensor digitorum longus muscles. J Neurophysiol 43:1615-1630.

Grillner S, Udo M (1971) Motor unit activity and stiffness of the contracting muscle fibres in the tonic stretch reflex. Acta Physiol Scand 81:422-424.

Gustafsson B, Pinter MJ (1985) On factors determining orderly recruitment of motor units - a role for intrinsic membrane-properties. Trends Neurosci 8:431-433.

Heckman CJ, Lee RH, Brownstone RM (2003) Hyperexcitable dendrites in motoneurons and their neuromodulatory control during motor behavior. Trends Neurosci 26:688-695.

Heckman CJ, Gorassini MA, Bennett DJ (2005) Persistent inward currents in motoneuron dendrites: implications for motor output. Muscle Nerve 31:135-156.

Henneman E, Somjen G, Carpenter DO (1965) Functional significance of cell size in spinal motoneurons. J Neurophysiol 28:560-580.

Hornby TG, McDonagh JC, Reinking RM, Stuart DG (2002) Motoneurons: a preferred firing range across vertebrate species? Muscle Nerve 25:632-648.

Huizar P, Kuno M, Miyata Y (1975) Electrophysiological properties of spinal motoneurones of normal and dystrophic mice. J Physiol 248:231-246.

Huizar P, Kuno M, Kudo N, Miyata Y (1977) Reaction of intact spinal motoneurones to partial denervation of the muscle. J Physiol 265:175-191.

Hultborn H, Brownstone RB, Toth TI, Gossard JP (2004) Key mechanisms for setting the input-output gain across the motoneuron pool. Prog Brain Res 143:77-95.

Iglesias C, Meunier C, Manuel M, Timofeeva Y, Delestrée N, Zytnicki D (2011) Mixed mode oscillations in mouse spinal motoneurons arise from a low excitability state. J Neurosci 31:5829-5840.

Kernell D (2006) The motoneurone and its muscle fibres. Oxford, UK: Oxford UP.

Kudina LP (1999) Analysis of firing behaviour of human motoneurones within 'subprimary range'. J Physiol Paris 93:115-123. 
Kudina LP, Alexeeva NL (1992) After-potentials and control of repetitive firing in human motoneurones. Electroencephalogr Clin Neurophysiol 85:345-353.

Manuel M, Meunier C, Donnet M, Zytnicki D (2006) The afterhyperpolarization conductance exerts the same control over the gain and variability of motoneurone firing in anaesthetized cats. J Physiol 576:873-886.

Manuel M, Meunier C, Donnet M, Zytnicki D (2007) Resonant or not, two amplification modes of proprioceptive inputs by persistent inward currents in spinal motoneurons. J Neurosci 27:12977-12988.

Manuel M, Iglesias C, Donnet M, Leroy F, Heckman CJ, Zytnicki D (2009) Fast kinetics, high-frequency oscillations, and subprimary firing range in adult mouse spinal motoneurons. J Neurosci 29:11246-11256.

Meehan CF, Sukiasyan N, Zhang M, Nielsen JB, Hultborn H (2010) Intrinsic properties of mouse lumbar motoneurons revealed by intracellular recording in vivo. J Neurophysiol 103:2599-2610.
Palmer SS, Fetz EE (1985) Discharge properties of primate forearm motor units during isometric muscle activity. J Neurophysiol 54:1178-1193.

Powers RK, Binder MD (2003) Persistent sodium and calcium currents in rat hypoglossal motoneurons. J Neurophysiol 89:615-624.

Schwindt PC, Crill WE (1980) Properties of a persistent inward current in normal and TEA-injected motoneurons. J Neurophysiol 43:1700-1724.

Stauffer EK, McDonagh JC, Hornby TG, Reinking RM, Stuart DG (2007) Historical reflections on the afterhyperpolarization-firing rate relation of vertebrate spinal neurons. J Comp Physiol A Neuroethol Sens Neural Behav Physiol 193:145-158.

Turkin VV, O’Neill D, Jung R, Iarkov A, Hamm TM (2010) Characteristics and organization of discharge properties in rat hindlimb motoneurons. J Neurophysiol 104:1549-1565.

Zengel JE, Reid SA, Sypert GW, Munson JB (1985) Membrane electrical properties and prediction of motor-unit type of medial gastrocnemius motoneurons in the cat. J Neurophysiol 53:1323-1344. 